\title{
Photoproduction of Charm Pairs
}

\author{
Erik E. Gottschalk ${ }^{1}$ \\ Fermilab, P.O. Box 500, Batavia, IL 60510, USA
}

\begin{abstract}
.
A large sample of events containing fully and partially reconstructed pairs of charmed $D$ mesons has been studied by the Fermilab photoproduction experiment FOCUS (FNAL-E831). Correlations between photoproduced $D$ and $\bar{D}$ mesons are used to study heavy quark production dynamics. Correlation results are presented for fully and partially reconstructed pairs of charmed $D$ mesons. The results are compared to Monte Carlo predictions based on a recent version of PYTHIA with default settings.
\end{abstract}

\section{INTRODUCTION}

A fundamental understanding of heavy-quark production has not yet been achieved. Quantum Chromodynamics (QCD) provides a theoretical framework, but perturbative QCD calculations can only be applied to some aspects of heavy-quark production while other aspects remain elusive. This is especially true for charm production, where perturbative QCD calculations have large uncertainties and non-perturbative effects must be included to adequately model physical observables. In the absence of a fundamental understanding of heavy-quark production, the best tools we have are models that are able to reproduce existing experimental data and make predictions about untested aspects of QCD.

One subject of considerable theoretical interest and ongoing experimental research is the study of correlations between heavy-quark pairs [1], in particular the study of correlations between $D$ and $\bar{D}$ mesons. In this paper, highlights from a recent publication [2] on $D \bar{D}$ correlations in photoproduction are presented. The results are based on studies of fully and partially reconstructed charm-pair events, and are presented by comparing data distributions to predictions from a recent version of the Lund model [3] for photon-gluon fusion. The overall agreement between our data and the model is good, and significant improvements have been made compared to older versions of the model $[4,5]$. In this paper we highlight noteworthy differences between our data and the model.

\footnotetext{
1

1 On behalf of the FOCUS Collaboration: J. M. Link, P. M. Yager (UC Davis); J. C. Anjos, I. Bediaga, C. Göbel, J. Magnin, A. Massafferri, J. M. de Miranda, I. M. Pepe, E. Polycarpo, A. C. dos Reis (CPBF, Rio de Janeiro); S. Carrillo, E. Casimiro, E. Cuautle, A. Sánchez-Hernández, C. Uribe, F. Vázquez (CINVESTAV, México City); L. Agostino, L. Cinquini, J. P. Cumalat, B. O’Reilly, I. Segoni, K. Stenson, M. Wahl (CU Boulder); J. N. Butler, H. W. K. Cheung, G. Chiodini, I. Gaines, P. H. Garbincius, L. A. Garren, E. E. Gottschalk, P. H. Kasper, A. E. Kreymer, R. Kutschke, M. Wang (Fermilab); L. Benussi, M. Bertani, S. Bianco, F. L. Fabbri, A. Zallo (INFN Frascati); M. Reyes (University of Guanajuato, Leon); C. Cawlfield, D. Y. Kim, A. Rahimi, J. Wiss (UI Champaign); R. Gardner, A. Kryemadhi (IU Bloomington); Y. S. Chung, J. S. Kang, B. R. Ko, J. W. Kwak, K. B. Lee (Korea University, Seoul); K. Cho, H. Park (Kyungpook National University, Taegu); G. Alimonti, S. Barberis, M. Boschini, A. Cerutti, P. D'Angelo, M. DiCorato, P. Dini, L. Edera, S. Erba, M. Giammarchi, P. Inzani, F. Leveraro, S. Malvezzi, D. Menasce, M. Mezzadri, L. Moroni, D. Pedrini, C. Pontoglio, F. Prelz, M. Rovere, S. Sala (INFN and Milano); T. F. Davenport III (UNC Asheville); V. Arena, G. Boca, G. Bonomi, G. Gianini, G. Liguori, D. Lopes Pegna, M. M. Merlo, D. Pantea, S. P. Ratti, C. Riccardi, P. Vitulo (INFN and Pavia); H. Hernandez, A. M. Lopez, E. Luiggi, H. Mendez, A. Paris, J. Quinones, J. E. Ramirez, Y. Zhang (Mayaguez, Puerto Rico); J. R. Wilson (USC Columbia); T. Handler, R. Mitchell (UT Knoxville); D. Engh, M. Hosack, W. E. Johns, M. Nehring, P. D. Sheldon, E. W. Vaandering, M. Webster (Vanderbilt); M. Sheaff (Wisconsin, Madison)
} 


\section{CHARM-PAIR ANALYSIS}

The data for our studies of photoproduced charm pairs were recorded by the FOCUS (FNAL-E831) experiment during the 1996-1997 fixed-target run at the Fermi National Accelerator Laboratory. The experiment ran with a photon beam and a $\mathrm{BeO}$ target. The average photon energy for the recorded data was $\approx 180 \mathrm{GeV}$ with a width of $\approx 50 \mathrm{GeV}$.

A candidate-driven algorithm [2] was used to collect a sample of $\approx 7000$ pairs of fully reconstructed $D$ mesons: $D^{+} D^{-}, D^{+} \bar{D}^{0}, D^{0} D^{-}$, and $D^{0} \bar{D}^{0}$. For this sample we considered the decay modes $D^{0} \rightarrow K^{-} \pi^{+}, D^{+} \rightarrow K^{-} \pi^{+} \pi^{+}, D^{0} \rightarrow K^{-} \pi^{+} \pi^{+} \pi^{-}$, and charged-conjugate modes. The algorithm found charm-pair events by reconstructing two $D$-meson candidates, and by using the two $D$ 's to reconstruct a primary vertex. The algorithm included other tracks in the primary vertex as long as the tracks satisfied confidence level cuts associated with the primary vertex. The number of tracks found in this manner together with the two $D$ 's define $N_{\text {primary }}$, the primary vertex multiplicity.

Figure 1 shows the $D \bar{D}$ signal that we obtain. The figure shows the normalized $D$ invariant $\operatorname{mass}^{2} M_{n}(D)$ opposite the normalized $\bar{D}$ invariant mass $M_{n}(\bar{D})$. The $D \bar{D}$ yield that we obtain from a fit to the data is $7064 \pm 119$ (statistical error).

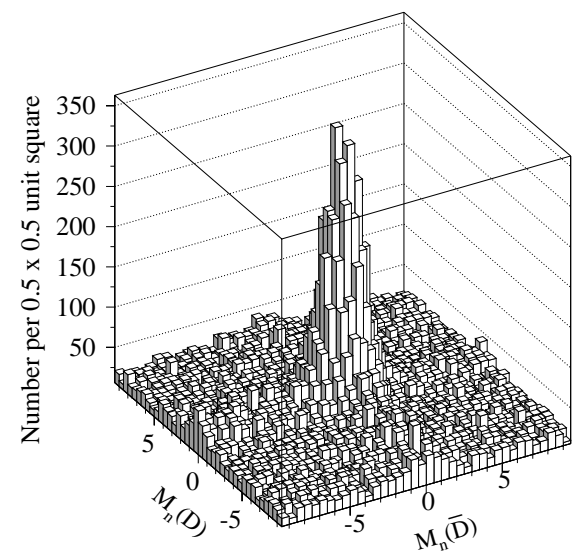

FIGURE 1. Normalized $D$ mass vs. normalized $\bar{D}$ mass distribution for fully reconstructed charm pairs.

In addition to our sample of pairs of fully reconstructed $D$ mesons, we have also obtained a sample of events where one $D$ is fully reconstructed (referred to as the recoil $D$ ) and the other is kinematically tagged by a slow pion coming from the decay $D^{*+} \rightarrow \pi^{+} D^{0}$. In these decays, the $D^{0}$ need not be reconstructed, and therefore we refer to this sample of events as partially reconstructed charm pairs ${ }^{3}$. For this sample we consider the same decay modes that were used for the fully reconstructed charm pairs. Figure 2a shows the invariant mass distribution that we obtain, with a total of $782630 \pm 1600$ candidates satisfying our selection criteria.

2 The normalized mass, $M_{n}(D)=\Delta M / \sigma_{M}$, is defined as the difference between the reconstructed mass and the central value of the $D^{+}$or $D^{0}$ mass distribution divided by the reconstructed-mass error $\sigma_{M}$.

${ }^{3}$ The partially reconstructed sample consists of $D^{*+} D^{-}, D^{*+} \bar{D}^{0}, D^{0} D^{*-}$, and $D^{+} D^{*-}$ pairs. 

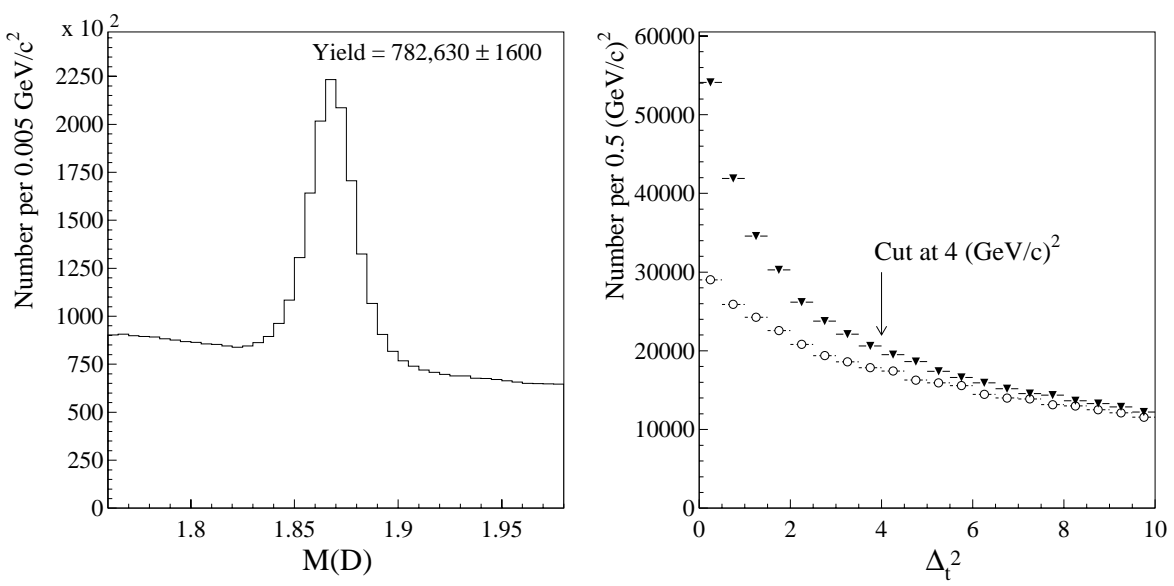

FIGURE 2. Invariant mass of the recoil $D$ in the partially reconstructed charm-pair sample (left). The mass of charged $D$ candidates is lowered by $3.74 \mathrm{MeV} / c^{2}$ to match the $D^{0}$ mass. The yield is a sum of individual yields for the three decay modes described in the text. $\Delta_{t}^{2}$ distributions (right) for right-sign (filled triangles) and wrong-sign (open circles) combinations of partially reconstructed charm pairs.

For partially reconstructed charm pairs we perform a background subtraction that is based on the charge of the slow pion. This is done by treating each track that is assigned to the primary vertex (excluding the recoil $D$ ) as a slow-pion candidate from the decay $D^{*+} \rightarrow \pi^{+} D^{0}$. If the charge of the slow pion is the same as the charge of the kaon from the recoil $D$, then the combination of the slow pion and recoil $D$ is designated as a rightsign combination. Otherwise, it is a wrong-sign combination. This is used to subtract wrong-sign background from right-sign combinations.

To enhance the event selection procedure, a maximum cut of $4(\mathrm{GeV} / c)^{2}$ is applied to $\Delta_{t}^{2}=\left(p_{x}^{(r)}+13.8 * p_{x}^{(\pi)}\right)^{2}+\left(p_{y}^{(r)}+13.8 * p_{y}^{(\pi)}\right)^{2}$, where $p_{x}^{(r)}, p_{y}^{(r)}$ and $p_{x}^{(\pi)}, p_{y}^{(\pi)}$ are transverse momentum components of the recoil $D$ and slow pion ${ }^{4}$, respectively. This cut enhances the selection of signal since genuine events balance $\Delta_{t}^{2}$ (see reference [5] for more details). This is shown in Figure 2b, which shows a prominent excess of right-sign combinations close to $\Delta_{t}^{2}=0$ compared to the wrong-sign background. After applying the $\Delta_{t}^{2}$ cut, we obtain a sample of $75160 \pm 1040$ partially-reconstructed charm pairs.

\section{CHARM-PAIR PRODUCTION}

Our study of charm-pair production compares FOCUS data to predictions from a Monte Carlo based on PYTHIA 6.203 [3] with default settings. We use PYTHIA with default settings (instead of using a Monte Carlo tuned to match our data) to facilitate comparisons with theoretical predictions and results from other experiments. To improve comparisons between data and model predictions we eliminate our lowest multiplicity

\footnotetext{
${ }^{4}$ The momentum of the soft pion approximates the momentum of the $D^{*}$ (due to the low $Q$ value of the $D^{*}$ decay) when multiplied by the inverse of its energy fraction, which is $\approx 13.8$.
} 
charm-pair events by requiring $N_{\text {primary }}>2$. This eliminates events that have only the two $D$ mesons assigned to the primary and no additional tracks. The reason for imposing this cut when comparing data to PYTHIA is that the cut removes diffractively produced $\psi(3770)$ events, which are not present in PYTHIA. $\psi(3770)$ events are observed in FOCUS data (see Figure 3 ) as a threshold enhancement in the invariant $D \bar{D}$ mass for mass combinations with a net charge of zero $\left(D^{+} D^{-}\right.$and $\left.D^{0} \bar{D}^{0}\right)$. This enhancement is especially evident for events with $N_{\text {primary }}=2$ when we apply cuts that remove events with energy deposited in our electromagnetic calorimeters (see Figure 3b).
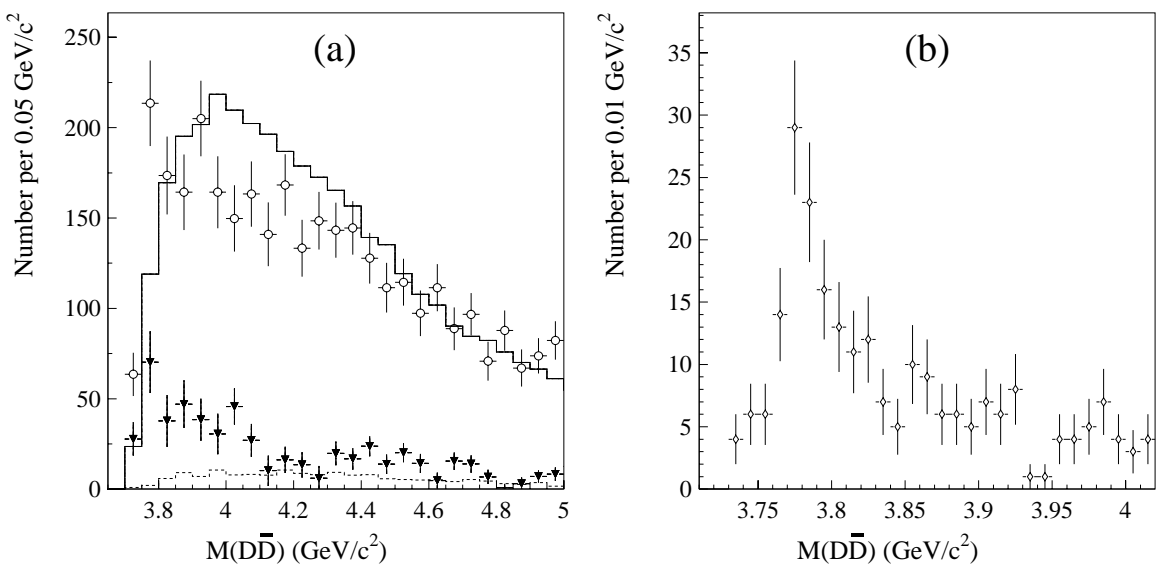

FIGURE 3. (a) Invariant $D \bar{D}$ mass for $D^{+} D^{-}$and $D^{0} \bar{D}^{0}$ mass combinations for background-subtracted FOCUS data (open circles), PYTHIA 6.203 (solid line), FOCUS data with an $N_{\text {primary }}=2$ cut applied to the data (filled triangles), and Pythia 6.203 with $N_{\text {primary }}=2$ cut (dashed line). (b) The $D \bar{D}$ mass for $N_{\text {primary }}=2$ after removing events with energy deposited in electromagnetic calorimeters.

After requiring $N_{\text {primary }}>2$ for fully reconstructed charm pairs, we compare FOCUS data to PYTHIA 6.203 for both the fully and partially reconstructed ${ }^{5}$ charm pairs (see Figures 4 and 5). Although we observe some discrepancies, the PYTHIA model for charm photoproduction shows good agreement with the data. The distribution for the azimuthal angle, $\Delta \phi$, between the $D$ and $\bar{D}$ momentum vectors in the plane transverse to the beam direction is reproduced by PYTHIA; however, we observe an enhancement in the first $\Delta \phi$ bin that may suggest the presence of a production mechanism that is not included in PYTHIA. The enhancement disappears for partially reconstructed charm pairs (see Figure 5a) due to resolution broadening and selection cuts used in the analysis. There is good agreement for the transverse momentum squared of the $D \bar{D}$ pair in Figures $4 \mathrm{~b}$ and $5 \mathrm{~b}$, except that the data tend to have slightly larger values of $p_{t}^{2}(D \bar{D})$. Other comparisons between FOCUS data and PYTHIA are presented in reference [2].

In summary, FOCUS has extracted two large samples of charm pairs for studies of charm photoproduction. The agreement between data and a recent version of PYTHIA with default settings is good. Noteworthy differences are observed in the azimuthal angle between the $D$ and $\bar{D}$ mesons, and in the production of $\psi(3770)$ events.

\footnotetext{
5 Partially reconstructed charm-pair events satisfy an implicit $N_{\text {primary }}>2$ cut, since these events have
} two $D$ mesons and a slow pion that account for a minimum primary vertex multiplicity of three. 

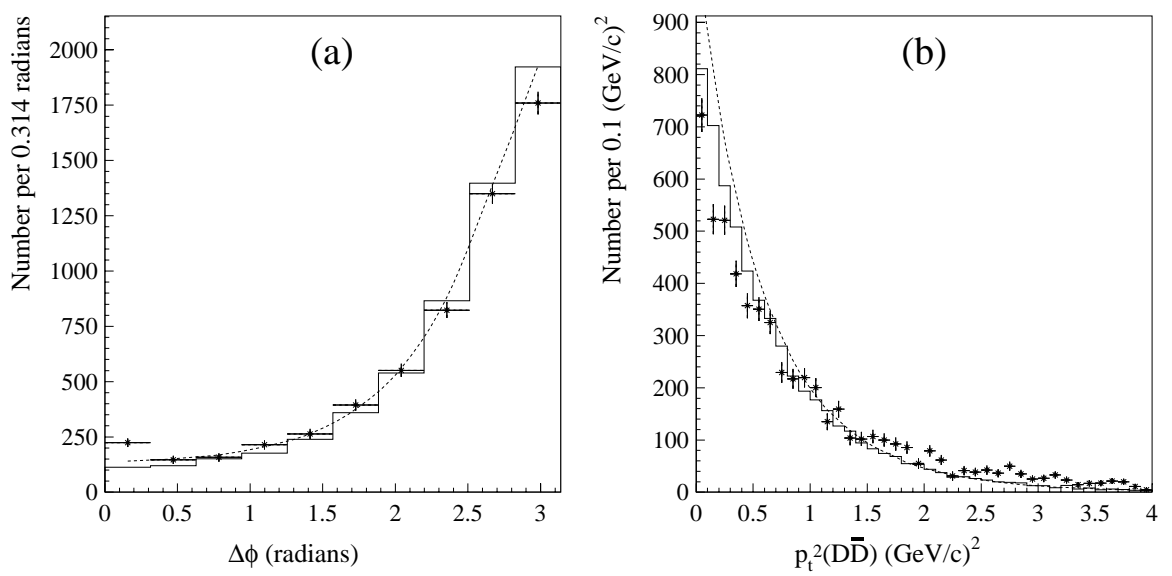

FIGURE 4. Charm-pair correlations for fully reconstructed charm pairs with $N_{\text {primary }}>2$ : (a) $\Delta \phi$ and (b) $p_{t}^{2}$ of the $D \bar{D}$ pair for background-subtracted FOCUS data (asterisks with error bars), PYTHIA 6.203 after detector simulation and data analysis cuts (solid line), and PYTHIA 6.203 parent distributions without acceptance or resolution effects (dashed line with arbitrary normalization).
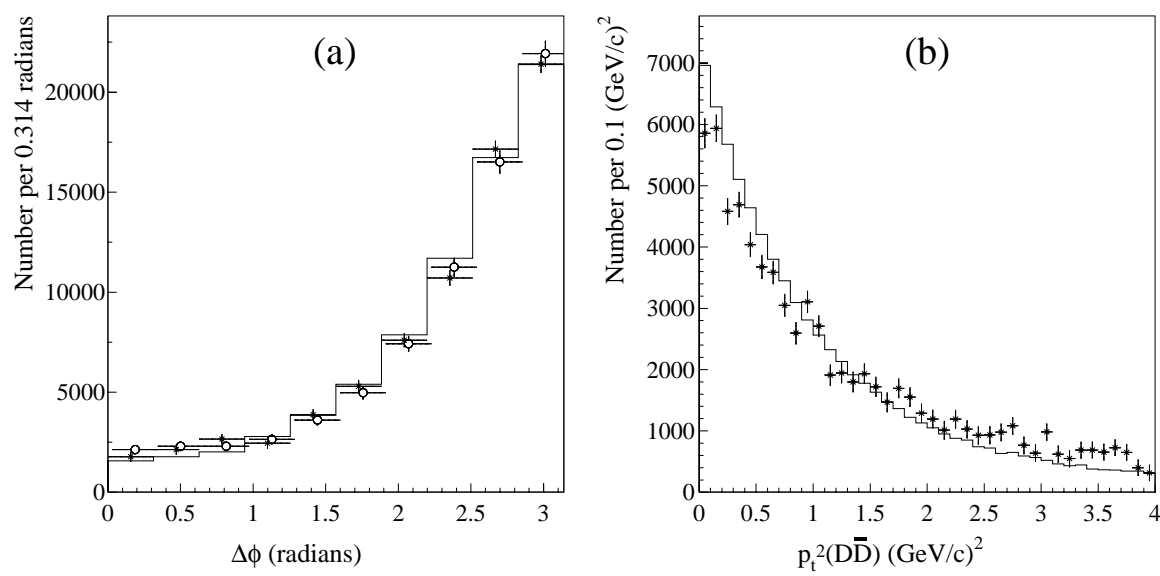

FIGURE 5. Charm-pair correlations for partially reconstructed charm pairs: (a) $\Delta \phi$ and (b) $p_{t}^{2}$ of the $D \bar{D}$ pair for background-subtracted FOCUS data (asterisks with error bars) and PYTHIA 6.203 after detector simulation and data analysis cuts (solid line). The $\Delta \phi$ distribution for fully-reconstructed charm pairs (open circles with error bars) is included for comparison in (a) after accounting for resolution broadening.

\section{REFERENCES}

1. S. Frixione, M.L. Mangano, P. Nason, and G. Ridolfi, Adv. Ser. Direct. High Energy Phys. 15 (1998) 609 [arXiv:hep-ph/9702287].

2. $\quad$ FOCUS Collaboration, J.M. Link et al., Phys. Lett. B 566 (2003) 51.

3. Pythia 6.203, T. Sjöstrand et al., Comput. Phys. Commun. 135 (2001) 238.

4. Pythia 5.6 and Jetset 7.3, T. Sjöstrand et al., Comput. Phys. Commun. 82 (1994) 74.

5. E687 Collaboration, P.L. Frabetti et al., Phys. Lett. B 308 (1993) 193. 